\title{
Mandibular Asimetri ve Asemptomatik Denekler Arasındaki Temporomandibular Eklemde Biyomekanik Karşılaştırmanın Modifiye Sonlu Elemanlar Modellerini Kullanarak Yapılması
}

\author{
The Biomechanical Comparison of The Temporomandibular Joint Between Mandibular \\ Asymmetry and Asymptomatic Subjects Using Modified Finite Element Models
}

\author{
Oğuz KAYABAŞı ${ }^{1}$ \\ ${ }^{1}$ Düzce Üniversitesi, Mühendislik Fakültesi, Biyomedikal Mühendisliği Bölümü, 81620 Konuralp, Düzce
}

$\ddot{O} \mathbf{z}$

Mandibular asimetri ve asemptomatik denekleri olan hastalar arasındaki temporomandibular eklemlerdeki (TME) gerilme dağılımlarının farklılıkları, modifiye üç boyutlu (3D) sonlu elemanlar (SE) modelleri kullanılarak karşılaştırıldı. 10 asemptomatik denek ve on mandibular asimetrik hasta sırasıyla Kontrol ve Vaka grubunu oluştulmuştur. Homojen olmayan malzeme özellikleri, bilgisayarlı tomografiye (CBCT) göre yeniden yapılandırılan mandibula ve maksilla SE modellerinde kullanılmıştır. Etkileşim yüzeyleri temas elemanları olarak işlem görmüştür. Kuvvetler ve sınır şartları, iki tıkanmaya karşılık gelen iki gruba uygulanmıştır. Simülasyon, olgu grubundaki merkezi ve ön oklüzyonlar altında TME'lerin gerilmesindeki sapma olmayan ve sapma tarafları arasındaki anlamlı farkları ortaya koymuştur $(\mathrm{p}<0.05)$. Vaka grubundaki von Mises gerilmeleri, özellikle sapmamış taraftaki Kontrol grubundakilerden anlamlı derecede daha yüksek oluştu. Hastaların TME'sinde anormal dağılımlar ve stres konsantrasyonu da bulunmuştur. Sonuç olarak, modifiye SE modelleri, maksillofasiyal sistemi simüle etmek için daha gerçekçi bir yol sağlamıştır. Ayrıca, mandibular asimetri, TME'nin gerilmesini artırabilir. Mandibular asimetrisi olan hastalarda TME'nin aşırı gerilmeleri temporomandibular bozukluklarla (TMD) ilişkili olduğu gözlemlendi.

Anahtar Kelimeler: Mandibular asimetri, Temporomandibular eklem (TME), Sonlu elemanlar yöntemi, Temporomandibular bozukluklar (TMD).

\begin{abstract}
The differences in stress distributions in the temporomandibular joints (TME) between patients with mandibular asymmetry and asymptomatic subjects were compared using modified three-dimensional (3D) finite element (SE) models. 10 asymptomatic subjects and ten mandibular asymmetric patients were formed the Control and Case group, respectively. Nonhomogeneous material properties were used in mandible and maxilla SE models, which were reconstructed according to computed tomography (CBCT). Interaction surfaces were treated as contact elements. Forces and boundary conditions were applied to two groups corresponding to two blockages. Simulation revealed significant differences between the deviation of the TMJs under the central and anterior occlusions in the case group and between the deviation sides $(\mathrm{p}<0.05)$. The stresses of von Mises in the case group were significantly higher than those in the Control group on the non-deviating side. Abnormal distributions and stress concentrations were also found in the TMJ of the patients. As a result, modified SE models provided a more realistic way to simulate the maxillofacial system. Also, mandibular asymmetry can increase the tension of the TMJ. In patients with mandibular asymmetry, overstress of TMJ was observed to be associated with temporomandibular disorders (TMD).

Keywords: Mandibular asymmetry, Temporomandibular joint (TMJ), Finite element method, Temporomandibular disorders (TMD).

\section{I.GİRIŞ}

Sadece insanın kafasındaki eklemler gibi temporomandibular eklemler (TME) günlük çiğneme, konuşma ve yutma üzerinde önemli bir etkiye sahiptir. Diğer eklemlerden farklı olarak, çalışma sırasında TME'ler bağlanmıştır. Farklı hareketler nedeniyle, tek TME öklüzyon ve mandibular deformiteden etkilenir. Mandibular asimetri\% 8.7 -\% 23.3 arasında değişen belirgin bir prevalansı olan yaygın bir maksillofasiyal deformitedir [1-4]. Mandibuler asimetriye eşlik eden anormal konfigürasyon ve temporomandibular bozukluklar (TMD) bulunmuştur [5-8]. TME'deki
\end{abstract}


morfolojik farklılıklar, asemptomatik denekler ile mandibular asimetrik hastalar arasında bulunmuştur $[6,7,9]$ ve erken dönem mandibular asimetriyi teşhis etmek için standart olarak düşünülebilir. Biyomekanik ortamdaki farklılıklar hafif mandibular asimetriyi tedavi etmek için önemli bir yaklaşım sağlayabilir. Bununla birlikte, ilgili biyomekanik analizin yetersizliği olmuştur.

Stomatolojide sonlu elemanlar (SE) analizi yoğun olarak geliştirilmiştir [10,11]. Oral bilim dalı olarak, TME'de SE yöntemleri de kullanılmıştır. Statik ve dinamik durumlarda sağlıklı bireyler için eklem disk veya eklem kıkırdağının stres ve yer değiştirme dağılımını simüle etmek için iki boyutlu FE modelleri kullanılmıştır[12,13]. Daha sonra, distraksiyon osteogenezinden önce ve sonra hastaların biyomekanik tepkisini elde etmek için 3D modeller geliştirilmiştir [14]. Ayrıca, asemptomatik denekler ve disk kayması olan hastalar için TME'nin kıkırdak dokularının biyomekanik davranışlarını araştırtırılmıştır [15]. Mandibular asimetrik hastalarda mekanik değişiklikler ile iç düzensizliğin prevalansı arasında belirgin bir ilişki vardır [16]. Bununla birlikte, yukarıdaki çalışmalar homojen malzemeler olarak mandibula ve maksilla olarak kabul edilmiştir. Hepimizin bildiği gibi, mandibula homojen olmayan malzemelere aitti ve boş alanı, spongiosis ve diğer yapıları homojen malzemelerle doğru şekilde simüle edilemedi [17]. Bu nedenle, kemik yapılarını simüle etmek için homojen olmayan malzeme özellikleri gereklidir.

Bu çalışmada asemptomatik bireylerin ve mandibular asimetrik hastaların biyomekanik davranışlarını karşılaştırmak amacıyla SE modellerini yeniden oluşturmak için homojen olmayan malzeme özellikleri kullanılmıştır. Ayrıca, TME'deki stres dağılımları ile TMD semptomları arasındaki ilişkiler incelenmiştir. Mandibular asimetri ve TMD için yeni bir tedavi stratejisi sağlayabilir.

\section{MATERYAL VE YÖNTEM}

Mandibular asimetri tanısı alan 10 asemptomatik denek (4 kadın, 6 erkek, 26,7 $\pm 4,8$ yaşında) ve 10 hasta (5 kadın ve 5 erkek, 24,6 \pm 4,8 yaşında) sırasıyla Kontrol ve Olgu grubu olarak görev yapmıștır. Daha önce TME ile ilişkili prosedürleri olmayan ve fasiyal orta hattan sapan $5 \mathrm{~mm}$ 'den fazla olan 18 yaşın üzerindeki hastalar bizim mandibula asimetrik hastaları olarak kabul edilmiştir. Sağlıklı bir fiziksel durumu olan, hiç TMD belirtisi olmayan, dejeneratif hastalık ve yüz simetrisine sahip olmayan kişiler önceden TME ile ilgili prosedürler olmadan Kontrol grubundan oluşuyordu. Ayrıca, klinik olgular TMD'li 7 hasta gösterdi ve bunların hepsinde TMJ klikası vardı, 5'inde eklem ağrısı, 6'sında eşzamanlı bilateral eklem hareketi ve 3'ünde ağız açıklığı sınırlıydı. Tüm bireylere yazılı bilgilendirilmiş onay verilmiş ve amaçlar için insan etik onayı alınmıştır. Solda mandibular sapma olan sekiz hasta vardı. Bir önceki çalışmaya göre [18], sağ ve sol taraf sapmış ve sapmamıș taraf olarak tanımlanmıștır. Kalan sağa mandibular sapma olan iki hasta, sağa mandibular sapma olan hastaların sol ve sağ tarafları sırasıyla, sapma tarafi ve sapma olmayan taraf olarak tanımlanmıştır.

Tüm SE modelleri, 400 x 400 piksel çözünürlükte CT'den (CBCT) elde edilmiştir. Her CBCT için dilim artışı 0.4 mm'dir. CBCT taramalarından mandibula, maksilla ve disk ayırt edildi ve diğer yapılardaki farklı gri değerlere göre $\mathrm{SE}$ modellerinde üretimiştir. $\mathrm{Bu}$ çalışmada, tüm kemik yapıları doğrusal elastik, izotropik homojen olmayan malzemeler olarak kabul edilmiştir. MIMICS'deki (Materialize, Leuven, Belgium) homojen olmayan malzeme özelliklerini simüle etmek için Young modülü ile Hounsfield birimleri arasındaki ilişkilere dayanan ampirik formüllerden 100 malzeme alınmıştır [19-23]. Poisson'ın disk ve kemik yapılarının oranı ABAQUS'ta (Dassault SIMULIA, ABD) sirasiyla 0.4 ve 0.3 olarak tanımlanmıştır. Etkileşen yüzeyler, 0,001 [24] sürtünme katsayısına sahip bir temas modu olarak işlenmiştir. Temas bölgelerinde C3D10M eleman tipi kullanılmıştır. Modellerin diğer yapıları için C3D4 eleman tipi kullanılmıştır. Tüm modeller yaklaşık 210.000 elemandan oluşmuştur.

İki grubun modellerine merkezi ve anterior oklüzyonlara karşılık gelen yükler yapılmıştır. Dokuz ana kas kuvvetinin yönleri ve değerleri önceki çalışmalardan elde edilmiştir [25-27]. Ve üst yüzeyin altı serbestlik derecesi sınırlandırılmıştır. SE modeli Şekil 1'de gösterilmektir.
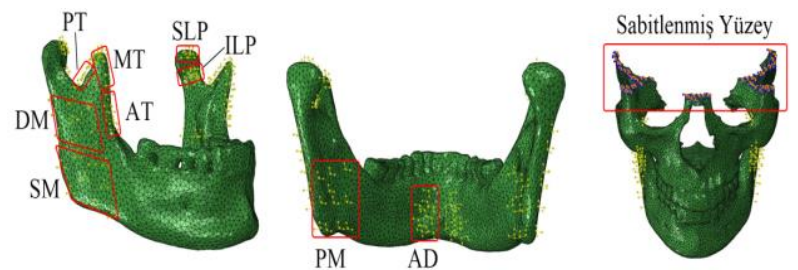

Şekil 1. Yükleme ve sınır şartları.

Çalışmamızda Von Mises gerilme seçilmiştir. Kontrol grubu için sol ve sağ taraflar arasında veya Durum grubu için sapma ve sapma olmayan taraflar arasındaki gerilimler sırasıyla eşleştirilmiş örneklem $t$ testi ile karşılaştırılmıştır. Ek olarak, Vaka ve Kontrol grupları arasındaki gerilmeler bağımsız örneklem t testi ile gerçekleştirilmiştir.

\section{BULGULAR}

Sapmış olmayan taraftaki TME'nin Von Mises gerilmesi, iki oklüzyon altında Olgu grubunun sapmış tarafindakilerden anlamlı derecede daha yüksek oluştu $(\mathrm{p}<0.05)$ (Tablo 1) (Şekil 2 ve Şekil 3). Bununla birlikte, Kontrol grubundaki TME'nin sol ve sağ tarafları arasındaki gerilme için iki oklüzyon altında anlamlı bir fark bulunmamıştır. Değerler Tablo 1'de gösterilmektedir. 
Tablo 1. Mandibular asimetri ve santral ile anterior oklüzyonlar altındaki asemptomatik deneklerde temporomandibular eklemde von Mises gerilmenin (MPa) ortalama ve standart sapmasi.

\begin{tabular}{|c|c|c|c|}
\hline Vaka & & $\begin{array}{c}\text { Sapma } \\
\text { olmayan } \\
\text { taraf }\end{array}$ & $\begin{array}{c}\text { Sapma } \\
\text { taraf }\end{array}$ \\
\hline \multirow{3}{*}{$\begin{array}{c}\text { Merkez } \\
\text { oklüzyon }\end{array}$} & Disk & $4.221 \pm 1.065$ & $3.055 \pm 1.378^{*}$ \\
\hline & Kondil & $9.669 \pm 2.659$ & $6.934 \pm 1.159^{*}$ \\
\hline & $\begin{array}{l}\text { Şakak } \\
\text { kemiği }\end{array}$ & $5.999 \pm 3.067$ & $3.724 \pm 1.516^{*}$ \\
\hline \multirow{3}{*}{$\begin{array}{l}\text { Anterior } \\
\text { oklüzyon }\end{array}$} & Disk & $\mathbf{3 . 5 3 4} \pm 1.473$ & $\underset{*}{2.379 \pm 0.899^{*}}$ \\
\hline & Kondil & $8.253 \pm 2.620$ & $6.628 \pm 2.050^{*}$ \\
\hline & $\begin{array}{l}\text { Şakak } \\
\text { kemiği }\end{array}$ & $5.452 \pm 3.400$ & $3.260 \pm 2.323^{*}$ \\
\hline Kontrol & & Sol taraf & Săg taraf \\
\hline
\end{tabular}
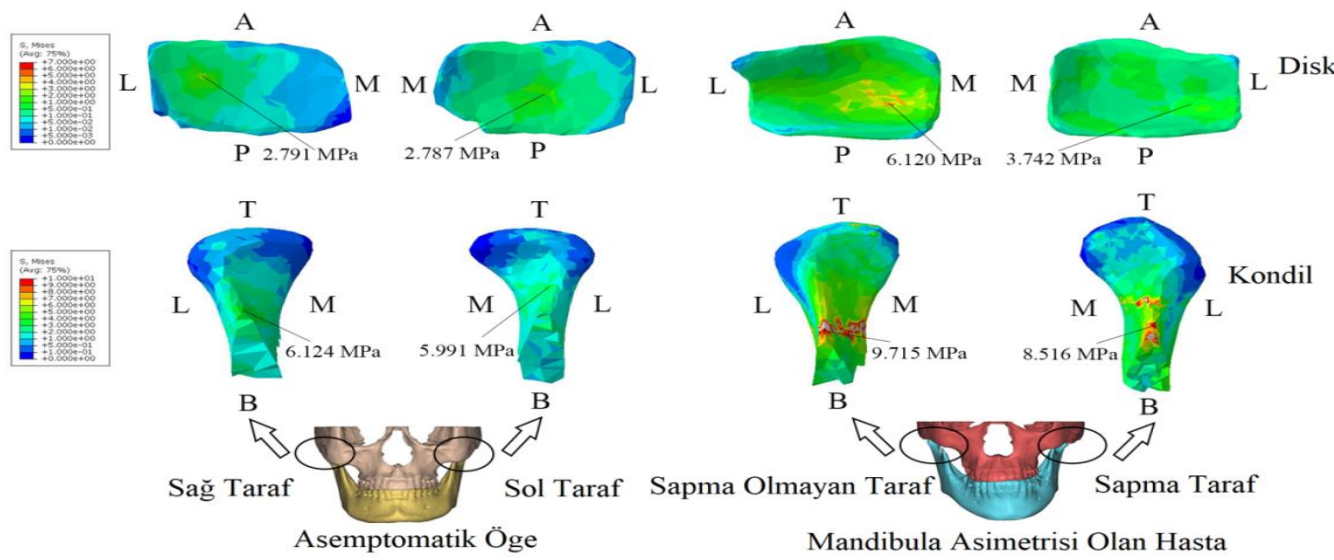

Şekil 2. von Mises, asemptomatik bir konunun ve merkezi tıkanıklık altındaki mandibular asimetrik bir hastanın kondillerinin disk ve gerilme dağılımlarını göstermektedir. A, ön; P, arka; M, medial; L, yanal; T, üst; B, alt.
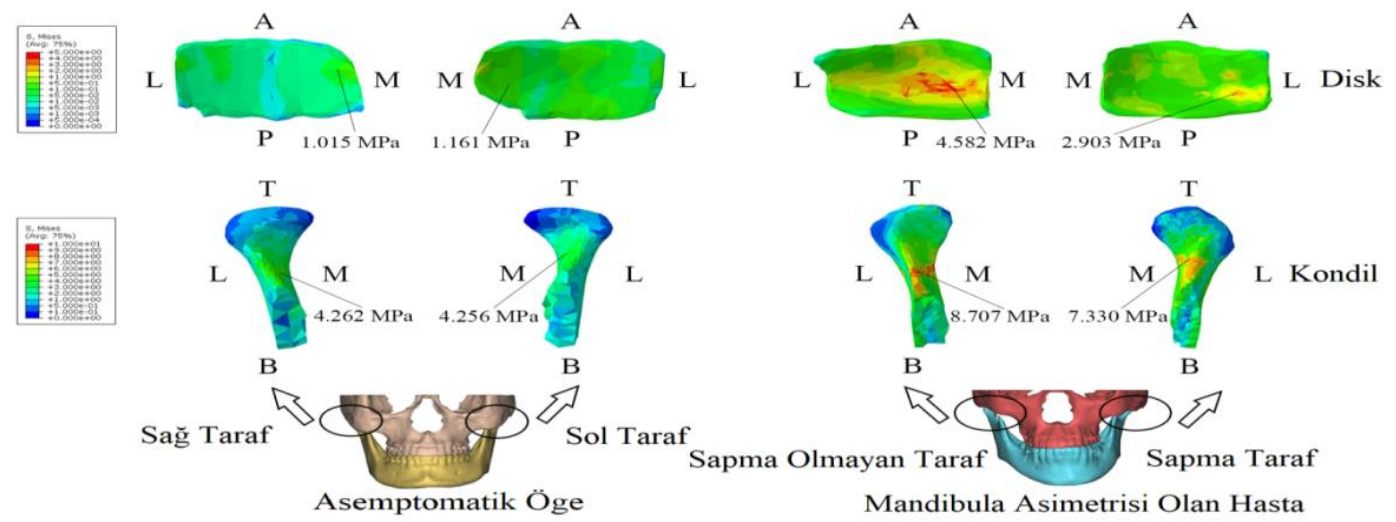

Şekil 3. von Mises, asemptomatik bir konunun ve ön oklüzyon altında bir mandibular asimetrik hastanın disklerinin ve kondillerinin gerilme dağılımlarını göstermektedir. A, ön; P, arka; M, medial; L, yanal; T, üst; B, alt. 
Simetrik gerilmeler nedeniyle, Tablo 2'de gösterilen Kontrol grubundaki gerilmelerin büyüklükleri her iki TME'nin ortalaması olarak oluşmuştur. Sonuçlar, Vaka grubundaki TME'lerin von Mises gerilmesi kontrol grubundakilerden daha yüksek olduğunu göstermiştir (Tablo 2). Vaka grubundaki TME'lerin sapma olmayan taraflarındaki gerilme, iki oklüzyon altındaki Kontrol grubundakilere göre anlamlı ya da yüksek derecede anlamlıydı (p <0.05). Vaka grubunun sapmış taraflarında, von Mises, Vaka grubu için disklerin stresleri, ön oklüzyon altındaki Kontrol grubundakilerden anlamlı derecede daha gerçekleşmiştir (Tablo 2).

Tablo 2. Merkez ve anterior oklüzyonlar altındaki çalışma ve kontrol grupları için emporomandibular eklemde von Mises gerilmesinin (MPa) ortalama ve standart sapmasi.

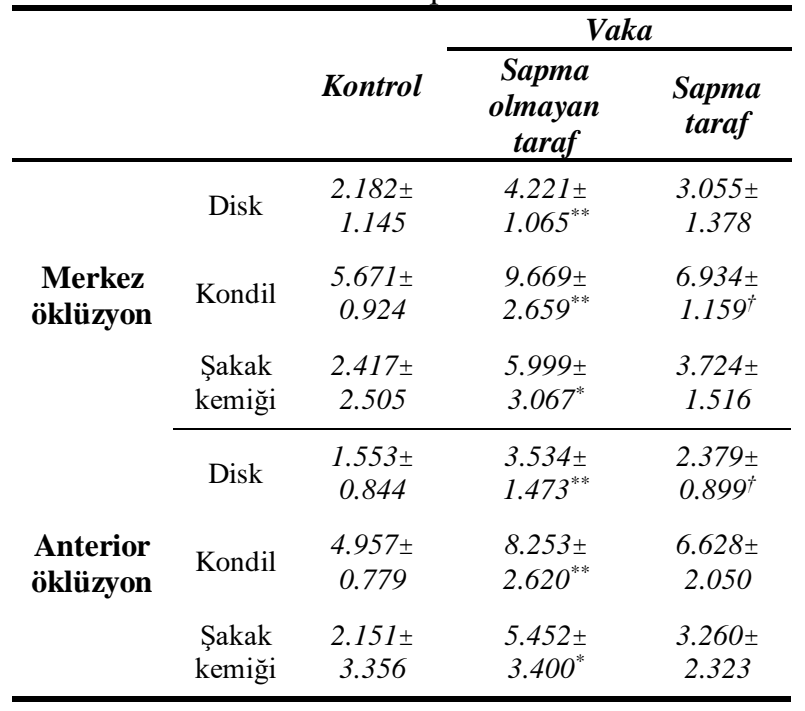

Not: Olgu: mandibula asimetrisi olan hastalar; Kontrol: normal konular. $\mathrm{p}>0.05$, anlamlı değil. * Olgu grubunun ve kontrol grubunun sapmayan tarafları arasındaki bağımsız örneklem $\mathrm{t}$ testi ile istatistiksel olarak anlamlı fark bulundu $(\mathrm{p}<0.05)$. ** Olgu grubunun sapmamış tarafi ile Kontrol grubu arasındaki bağımsız örneklem t-testi ile istatistiksel olarak anlaml fark. $(\mathrm{P}<0.01)$. † Bağımsız örneklem t-testi ile vaka grubunun ve kontrol grubunun sapmış tarafi arasındaki istatistiksel olarak anlamlı fark $(\mathrm{p}<0.05) . \dagger \dagger$ Bağımsız örneklem t-testi ile olgu grubunun ve Kontrol grubunun sapmış tarafı arasında istatistiksel olarak anlamlı fark. $(\mathrm{P}<0.01)$.

TME'lerin von Mises gerilme dağılımlarına gelince, maksimum von Mises gerilmeleri, kontrol grubundaki iki tıkanıklık altında esas olarak diskin orta bölgesinde bulunmuştur. Ancak, hastalar için diskin maksimum von Mises gerilme dağılımları Kontrol grubundakilerden farklı oluşmuştur (Tablo3).
Tablo 3. Vaka grubunda diskteki maksimum von Mises stresi yerleri.

\begin{tabular}{|c|c|c|c|c|}
\hline & & $\begin{array}{r}\text { Orta } \\
\text { bölge }\end{array}$ & $\begin{array}{l}\text { Posterior } \\
\text { bölge }\end{array}$ & $\begin{array}{c}\text { Anterior } \\
\text { bölge }\end{array}$ \\
\hline \multirow[t]{2}{*}{ Disk } & $\begin{array}{c}\text { Sapma } \\
\text { olmayan } \\
\text { taraf }\end{array}$ & 6 & 4 & 0 \\
\hline & $\begin{array}{c}\text { Sapma } \\
\text { taraf }\end{array}$ & 6 & 2 & 2 \\
\hline
\end{tabular}

\section{TARTIŞMA}

Önceki çalışmalar, mandibular asimetrisi olan hastalarda TME'nin morfolojisi ve konumlarının normal deneklerden farklı olduğunu doğrulamıştır $[5,6]$. Bununla birlikte, normal denekler ve hafif belirtileri ve semptomları iyileştirmede altın kriter haline gelebilecek olan TME'nin gerilme dağılımındaki mandibuler asimetrik hastalar arasındaki biyomekanik farklılıklar hala net değildir. Bu nedenle, TME'deki gerilme dağılımlarının mandibular asimetrisi olan hastalar ve normal denekler arasındaki farkları analiz etmek için gerekliydi.

Maksillofasiyal modeller önceki çalışmalarda her zaman homojen malzemeler olarak düşünülmüştür [28,29]. Çalışmamızda karmaşık TME yapısını simüle etmek için tek bir malzeme özelliğini kullanmak yanlış oldu. Gri değer ile tanımlanan malzeme özelliklerinin kemiğin simülasyonunda oldukça doğru olduğu gösterilmiştir. Gri değere ve Hounsfield ünitelerine dayanan ampirik ilişkiler, farklı Young modüllerini TME'deki homojen olmayan kemik yapıları simüle etmek için atamak için kullanılmıştır [19]. Mandibula ve maksillaya, sırasıyla ilgili formüllere göre 100 farklı malzeme atanmıştır. Spongiosis ve boş alanlar tek malzeme özelliklerinde genellikle göz ardı edilmiş, homojen olmayan malzemeler kemik yapısını simüle etmek için daha doğru bir yol sağlanmıştır [17]. Ek olarak, disk ve kondil arasındaki, disk ve geçici kemikler arasındaki ve üst ve alt dişler arasındaki yüzeylerin, 0.001 sürtünme katsayısı ile temas ettiği kabul edilmiştir. 3D baskı modellerinin deneyleri, TME yapılarındaki etkileşimlerin simülasyonu için sürtünmeli temasin makul ve güvenilir olduğunu doğrulamıştır [30]. Bu nedenle, değiştirilmiş modeller çalışmamızda daha gerçekçi bir simülasyon sunmuştur.

Kontrol grubu için her iki TME arasında simetrik gerilme dağılımları karşılaştırıldığında, TME'nin gerilme dağılımları, merkezi tıkanma veya ön tıkanma ne olursa olsun, mandibular asimetrik hastalar için anlamlı derecede asimetrik olmuştur (Tablo 1). Sonuçlar, asimetrik geometrinin, TMJ'nin asimetrik stres dağılımına yol açacağını göstermiştir. Bu arada, von Mises TMJ'nin sapmamış tarafindaki stresler sapmış taraftakilerden önemli ölçüde daha büyüktü. TME'nin, eklemin sapma tarafında ciddi sıkışmalara yol açan hastalar için sapma olmayan tarafta daha fazla 
gerginlik ve kompresyona sahip olduğu açıktı. TMJ'nin bu asimetrik stres dağılımları, TMJ'nin uyumsuz fizyolojik fonksiyonlarına katkıda bulunmuştur. Klinik olgu kayıtlarında sınırlı ağız açıklığı (10 hastanın 3'ü) ve eşzamanlı bilateral eklem hareketi (10 hastanın 6'sı) bulundu.

Vaka grubundaki TME'lerin ortalama en yüksek von Mises gerilmeleri, Kontrol grubundakilerden açıç̧a daha büyüktür. TME'deki tüm parçaların von Mises gerilmelerinde, Vaka grubundaki ve Kontrol grubundaki iki taraf arasında sapma olmayan taraf arasında anlamlı farklılıklar vard1. $\mathrm{Bu}$ sonuçlar, mandibular asimetrinin, sapmamış yan TME'lerin gerilme seviyesini açıkça arttırabileceğini göstermiştir. Stresin artması ayrıca hastaların disklerinde ciddi sıkışmalara neden olmuştur. Önceki çalışmalar, mandibula asimetrisinin TME eklem alanlarını azaltacağını ve ayrıca eklem ağrısına, tıklamaya, disk perforasyonuna ve diğer TMD semptomlarına neden olacağını göstermiştir [9,31,32]. Bu çalışmada, bu çalışmaya alınan hastaların vaka kayıtları da oklüzyon tanısı alan 7 hasta ve eklem ağrılı 5 hasta gösterdi. T1klama ve eklem ağrısını öne süren sonuçlar, TME'lerin aşırı stresi ile de ilişkiliydi. Stresi azaltmak ve tıkanıklığı arttırmak için oklüzal atel ile eklem boşluklarını artırmak, eklem ağrısını iyileştirmede ve ciddi çene-yüz deformasyonu olmayan hastalarda kliklenmenin uygun olduğunu göstermiştir.

Mandibular asimetri, sadece asimetrik ve arttırılmış pik streslere yol açmaz, aynı zamanda TME'de anormal stres konsantrasyonuna neden olur. Daha önceki sonlu elemanlar çalışmasında von Mises gerilmesinin en büyük değerlerinin Kontrol grubumuzda olduğu gibi normal denekler için orta bölgeye yerleştirildiği görülmüş̧ür [33]. Bununla birlikte, posterior disk band1 her zaman düşük verim stresi için diskin en zayıf kısmı olarak kabul edilmiştir [34]. 4 hastanın sapma olmayan diskleri ve 2 hastanın sapma diskleri için arka disk bantı maksimum gerilmeyi sürdürmüştür. Hastalar için anormal pik gerilme de TME'nin diğer semptomları için potansiyel bir risk faktörüdür. Zamanında iyileşmezse, daha fazla TMD belirtisi yavaş yavaş ortaya çıkacaktır. Ayrıca, gerilme yoğunluğu hastalarda, özellikle kondil ve disk için zararlı olanlarda da bulunmuştur. Kondiler rezorpsiyon ve disk incelmesi riskini yükseltmiştir [30]. Bu nedenle, mandibular asimetri için ileri işlem, asimetrik gerilme dağılımlarını düzeltmeli, gerilme seviyesini düşürmeli, anormal tepe gerilmesini iyileştirmeli ve TME'deki stres konsantrasyonunu ortadan kaldırmalıdır.

\section{V.SONUÇLAR}

Homojen olmayan özellikleri simüle etmek için her yapı için 100 farklı malzeme içeren modifiye SE modelleri, çene-yüz sistemi modellemesi için daha gerçekçi bir yol sağlar. Modifiye FSE modelleri ile mandibular asimetrisi olan hastalar için her iki TME'de asimetrik gerilme dağılımları bulunmuştur. Ayrıca,
TMD semptomları ile ilişkili olarak, semptomsuz subjelere kıyasla TME gerilmesi de artabilir.

\section{KAYNAKLAR}

[1] Bergersen EO (1980) Enlargement and distortion in cephalometric radiography compensation tables for linear measurements. Angle Orthodontist 50:230-244

[2] Bishara SE, Burkey PS, Kharouf JG (1994) Dental and facial asymmetries - a review. Angle Orthodontist 64:89-98

[3] Ramirez-Yanez GO, Stewart A, Franken E, Campos K (2011) Prevalence of mandibular asymmetries in growing patients. European journal of orthodontics 33:236-242

[4] Haraguchi S, Iguchi Y, Takada K (2008) Asymmetry of the face in orthodontic patients. The Angle orthodontist 78:421-426

[5] Goncalves JR, Wolford LM, Cassano DS, da Porciuncula G, Paniagua B, Cevidanes LH (2013) Temporomandibular joint condylar changes following maxillomandibular advancement and articular disc repositioning. Journal of oral and maxillofacial surgery : official journal of the American Association of Oral and Maxillofacial Surgeons 71:1759 e1751-1715. doi:10.1016/j.joms.2013.06.209

[6] Ueki K, Moroi A, Sotobori M, Ishihara Y, Marukawa K, Yoshizawa K, Kato K, Kawashiri S (2012) Changes in temporomandibular joint and ramus after sagittal split ramus osteotomy in mandibular prognathism patients with and without asymmetry. The Journal of craniofacial surgery 40:821-827. doi:10.1016/j.jcms.2012.03.003

[7] Sanromán JF, González JMG, del Hoyo JA (1998) Relationship between condylar position, dentofacial deformity and temporomandibular joint dysfunction: An mri and ct prospective study. The Journal of craniofacial surgery 26:35-42

[8] Ueki K, Nakagawa K, Marukawa K, Takatsuka S, Yamamoto E (2005) The relationship between temporomandibular joint disc morphology and stress angulation in skeletal Class III patients. European journal of orthodontics 27:501-506. doi:10.1093/ejo/cji029

[9] Yang HJ, Hwang SJ (2014) Change in condylar position in posterior bending osteotomy minimizing condylar torque in BSSRO for facial asymmetry. The Journal of craniofacial surgery 42:325-332. doi:10.1016/j.jcms.2013.05.021

[10] 1Liu Z, Qian Y, Liu D, Yang J, Fan Y (2010) Stress analysis of first permanent mandibular molar with class 1 restorations of different cement bases by occlusive load: A finite element analysis. International Journal for Numerical Methods in Biomedical Engineering 
26:1371-1379. doi:10.1002/cnm.1395

[11] Qian Y, Liu Z, Fan Y (2010) Numerical simulation of canine bodily movement. International Journal for Numerical Methods in Biomedical Engineering:n/a-n/a. doi:10.1002/cnm.1179

[12] Chen J, Akyuz U, Xu L, Pidaparti RMV (1998) Stress analysis of the human temporomandibular joint. Medical engineering \& physics 20:565-572

[13] Qihong L, Shuang R, Cheng G, Haiyan S, Hong L, Yinzhong D, Qiguo R (2014) Effect of jaw opening on the stress pattern in a normal human articular disc: finite element analysis based on MRI images. Head \& face medicine 10

[14] Reina-Romo E, Sampietro-Fuentes A, GomezBenito MJ, Dominguez J, Doblare M, GarciaAznar JM (2010) Biomechanical response of a mandible in a patient affected with hemifacial microsomia before and after distraction osteogenesis. Medical engineering \& physics 32:860-866.

doi:10.1016/j.medengphy.2010.05.012

[15] Abe S, Kawano F, Kohge K, Kawaoka T, Ueda K, Hattori-Hara E, Mori H, Kuroda S, Tanaka E (2013) Stress analysis in human temporomandibular joint affected by anterior disc displacement during prolonged clenching. Journal of oral rehabilitation 50:230-244

[16] Buranastidporn B, Hisano M, Soma K (2006) Effect of biomechanical disturbance of the temporomandibular joint on the prevalence of internal derangement in mandibular asymmetry. European journal of orthodontics 28:199-205

[17] Kopp S, Kuzelka J, Goldmann T, Himmlova L, Ihde S (2011) Modeling of load transmission and distribution of deformation energy before and after healing of basal dental implants in the human mandible. Biomedizinische Technik Biomedical engineering 56:53-58.

doi:10.1515/BMT.2010.053

[18] 18. Ueki K, Nakagawa K, Takatsuka S, Shimada M, Marukawa K, Takazakura D, Yamamoto E (2000) Temporomandibular joint morphology and disc position in skeletal class III patients. The Journal of craniofacial surgery 28:362-368. doi:10.1054/jcms.2000.0181

[19] Shu JH, Yao J, Zhang YL, Chong DYR, Liu Z (2018) The influence of bilateral sagittal split ramus osteotomy on the stress distributions in the temporomandibular joints of the patients with facial asymmetry under symmetric occlusions. Medicine 97:e11204. doi:10.1097/MD.0000000000011204

[20] Ciarelli MJ, Goldstein SA, Kuhn JL, Cody DD, Brown MB (1991) Evaluation of orthogonal mechanical properties and density of human trabecular bone from the major metaphyseal regions with materials testing and computed tomography. J Orthop Res 9:674-682

[21] Harp JH, Aronson J, Hollis M (1994) Noninvasive determination of bone stiffness for distraction osteogenesis by quantitative computed tomography scans. Clin Orthop Relat Res:42-48

[22] Kopperdahl DL, Morgan EF, Keaveny TM (2002) Quantitative computed tomography estimates of the mechanical properties of human vertebral trabecular bone. J Orthop Res 20:801-805

[23] Rho JY, Hobatho MC, Ashman RB (1995) Relations of mechanical properties to density and ct numbers in human bone. Medical engineering \& physics 17:347-355

[24 Liu Z, Fan Y, Qian Y (2008) Comparative evaluation on three-dimensional finite element models of the temporomandibular joint. Clinical biomechanics 23 Suppl 1:S53-58. doi:10.1016/j.clinbiomech.2007.12.011

[25] Korioth TW, Hannam AG (1994) Deformation of the human mandible during simulated tooth clenching. Journal of dental research 73:56-66

[26] Pruim GJ, de Jongh HJ, ten Bosch JJ (1980) Forces acting on the mandible during bilateral static bite at different bite force levels. Journal of biomechanics 13:755-763

[27] Weijs WA, Hillen B (1984) Relationship between the physiological cross-section of the human jaw muscles and their cross-sectional area in computer tomograms. Acta Anatomica 118:129-138

[28] Liu Z, Qian YL, Zhang YL, Fan YB (2016) Effects of several temporomandibular disorders on the stress distributions of temporomandibular joint: A finite element analysis. Computer Methods in Biomechanics and Biomedical Engineering 19:137-143

[29] Qi XD, Ma LM, Zhong SZ (2012) The influence of the closing and opening muscle groups of jaw condyle biomechanics after mandible bilateral sagittal split ramus osteotomy. The Journal of craniofacial surgery 40:e159-164. doi:10.1016/j.jcms.2011.07.024

[30] Zhang YL (2017) The Effect of Bilateral Sagittal Split Ramus Osteotomy on Temporomandibular Joints in Patients with Facial Asymmetry: a Morphologic and Biomechanical Study. PhD Thesis, Sichuan University, Chengdu

[31] Zhang Y, Xu X, Liu Z (2017) Comparison of Morphologic Parameters of Temporomandibular Joint for Asymptomatic Subjects Using the Two-Dimensional and Three-Dimensional Measuring Methods. Journal of Healthcare Engineering 2017:1-8. doi:10.1155/2017/5680708

[32] Ueki K, Yoshizawa K, Moroi A, Iguchi R, Kosaka A, Ikawa H, Saida Y, Hotta A, Tsutsui 
$\mathrm{T}$ (2015) Changes in computed tomography values of mandibular condyle and temporomandibular joint disc position after sagittal split ramus osteotomy. The Journal of craniofacial surgery 43:1208-1217. doi:10.1016/j.jcms.2015.05.007

[33] Beek M, Koolstra JH, van Ruijven LJ, van Eijden TMGJ (2000) Three-dimensional finite element analysis of the human temporomandibular joint disc. Journal of biomechanics 33:307-316

[34 Kang H, Bao GJ, Qi SN (2006) Biomechanical responses of human temporomandibular joint disc under tension and compression. International journal of oral and maxillofacial surgery $35: 817-821$.

doi:10.1016/j.ijom.2006.03.005 